\title{
A Standard Matrix Assembly Approach for Static Analysis of Planar Reciprocal Structures
}

\author{
Ding Zou and Nan Xiao
}

\begin{abstract}
With the advantages of light weight and fast assembly, the reciprocal structures are promising in engineering application. Analytically to investigate the mechanical behavior of reciprocal structures is a challenge in practical designing due to interdependency of structural elements. Although static formulations for components could easily be established, their matrix assembly for an entire structure becomes a bottleneck because of non-hierarchical relationship between components. Different structural types may be decomposed into homogenous basic units. In order to unify the static calculation method for these structures, a standard format for matrix assembly is proposed and mathematical relationships between matrices of a basic unit and whole structure are established, aiming to facilitate the analysis and design of reciprocal structures, especially in large scale. A simple case study is shown to validate the process consistent with computational result. The proposed method may also apply to the analysis of any repetitive pattern for structural application.
\end{abstract}

Index Terms-Matrix assembly, planar, reciprocal structures, static analysis.

\section{INTRODUCTION}

Planar reciprocal structures had been found used for architectures in ancient time, such as the famous 'Serlio's Floor' [1] and 'Abeille's Bond' [2]. A model in Fig. 1 shows one possible configuration. In the last two decades, many studies focused on the configuration and constructability of reciprocal structures without much emphasis on mechanical analysis [3]-[6]. Although recently a few of research efforts have been conducted on mechanical behaviors of reciprocal structures [7], analytical methods are still in infancy due to the prevalence of finite element analysis [8]-[10] as well as the complex geometrical composition which significantly enhances difficulty of mathematical modelling. Since indeterminate structures require large calculating quantity, determinate systems would be a good starting point for mechanically characteristic analysis of reciprocal structures. A notable work has been initiated by Kohlhammer and Kotnik [11], however, the number of calculating in their method is surprisingly huge for large-scale structures. Another work investigated by Gelez et al. [12] aims only at one particular type of reciprocal structures with a changeable size, limitation is there for structures other than the specified by their analytical methods. This paper aims to develop a general approach that enables static formulation suggested by Kohlhammer \& Kotnik [11] to be efficient for

Manuscript received July 30, 2016; revised October 21, 2016

Ding Zou and Nan Xiao are with the College of Civil Engineering and Architecture, Zhejiang University, 310058 Hangzhou, China (e-mail: aa7onz@163,com, sholran@zju.edu.cn). a variety of planar reciprocal systems in large scale, extending the theory to broader application.

An obvious constitutive characteristic of reciprocal structures are that any node is joined with only two bars, and hence the force distribution at the node is simpler than that at a multi-bar joint in other commonly seen structures. In most cases, planar reciprocal structures mainly subject to vertical loads while horizontal ones can be ignorable with adequate boundary constraints. Therefore, only vertical loading will be considered in the following analysis.

Furthermore, most reciprocal structures, as a whole, can be decomposed into homogenous or heterogeneous basic units which consist of single bars enclosed together. In other words, three hierarchical levels of composition for reciprocal structures can be identified, i.e. whole system, basic unit and single bar.

To investigate the internal force under loading, analysis will start from a single bar and then the basic unit, finally the whole structure.

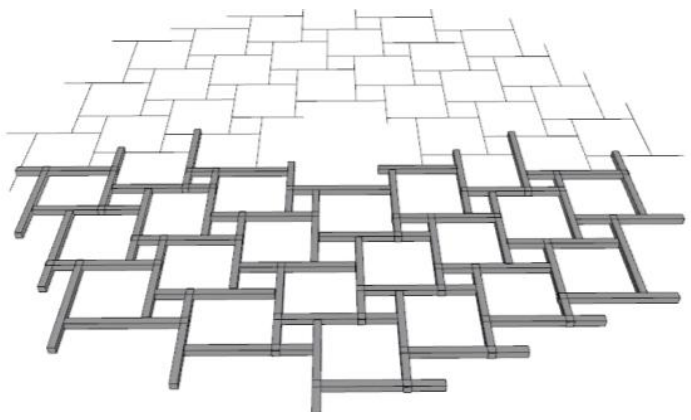

Fig. 1. Planar reciprocal structure.

\section{Static AnAlysis}

\section{A. Structural Characteristics}

Considering the pin-joint reciprocal structure in a horizontal plane subjected to vertical loading, every single bar acts as a simple beam and thus the entire structure is statically determinate, so that the internal force is only associated with the geometrical and topological relationship within the structure, free from material and bar cross section properties.

The mechanical behavior of reciprocal structures can be partially revealed by the analysis of a basic unit. For example, a three-bar unit which is the simplest form of the kind is pinned in the periphery (Fig. 2a). When a joint load is applied at node 4, it firstly distributes along the bar in opposite directions, one portion to the outer bearing and the other to the internal node 6 , their values depend on the position of the load on the bar. Similar distribution occurs at 
bar 3 and bar 2 displayed in Fig. 2b, resulting in part of the load comes back to node 4 and follows the same scheme again until the structure reaches its equilibrium. Now we can conclude that two types of force distribution reside in reciprocal structures, one is the circulation within the unit and the other the transmission between neighboring units which spreads the loads outwards. Also, the interdependency of single bars in the unit, or basic units in the whole system, makes reciprocal system structurally nonhierarchical.

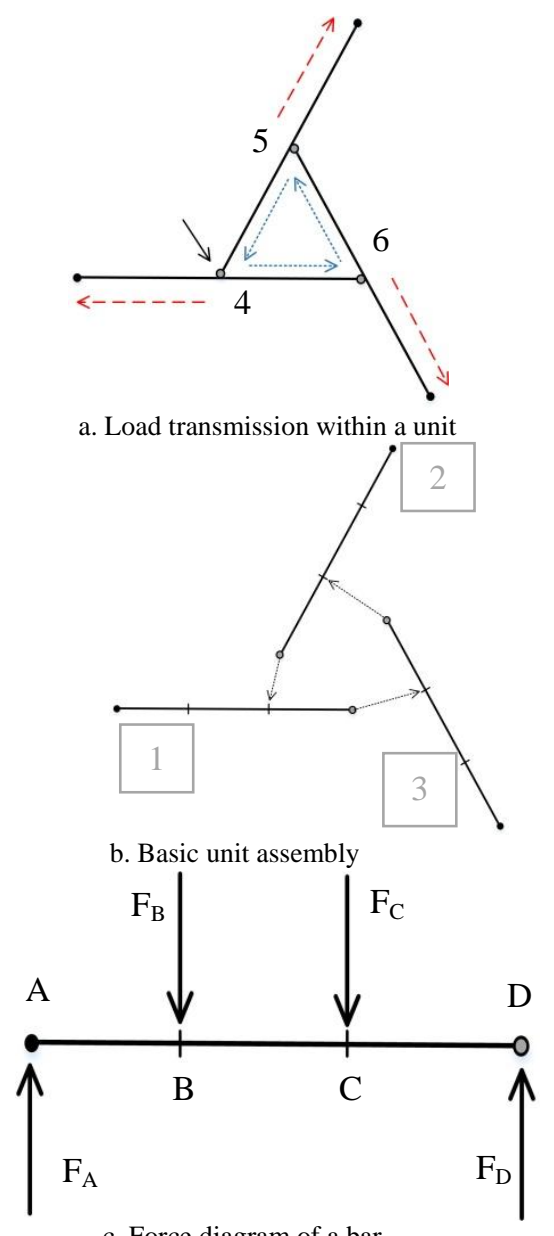

c. Force diagram of a bar

Fig. 2. Three-bar basic unit model.

\section{B. Static Formulation}

Following the qualitative description above, a quantitative analysis to acquire nodal forces by iterative mathematical formulation can be found in previous work [11]. Assuming all bars have identical condition of two loading points and two supporting ends (Fig. 2c), the nodal force at the $i$ th bar distribution can be expressed as

$$
\mathbf{F}_{i}=\left(\begin{array}{l}
F_{A} \\
F_{B} \\
F_{C} \\
F_{D}
\end{array}\right)_{i}=\left[\begin{array}{llll}
0 & c & a & 0 \\
0 & 0 & 0 & 0 \\
0 & 0 & 0 & 0 \\
0 & d & b & 0
\end{array}\right]\left(\begin{array}{l}
F_{A} \\
F_{B} \\
F_{C} \\
F_{D}
\end{array}\right)_{i-1}=\mathbf{A}_{b} \mathbf{F}_{i-1},
$$

where in the load distribution matrix $\mathbf{A}_{\mathrm{b}}$, a, c represent the load distribution coefficient from point $\mathrm{B}$ to point $\mathrm{A}$ and point $\mathrm{C}$ to point $\mathrm{A}$ respectively, and $\mathrm{b}, \mathrm{d}$ represent that from point $B$ to point $D$ and point $C$ to point $D$. Note that $a+b=c$ $+\mathrm{d}=1$, so there applies $\|\mathbf{A}\|<1$. The nodal force in equilibrium is the sum of that in each distribution step, formulated as

$$
\mathbf{F}=\sum_{i=0}^{\infty} \mathbf{F}_{i}=\left(\sum_{i=0}^{\infty} \mathbf{A}_{b}^{i}\right) \mathbf{F}_{0}=\left(\mathbf{I}-\mathbf{A}_{b}\right)^{-1} \mathbf{F}_{0}
$$

where $\mathbf{I}$ is the identity matrix with the same dimension of $\mathbf{A}_{\mathrm{b}}$ and $\mathbf{F}_{0}$ the initial load vector. For a three-bar basic unit, the load distribution matrix is initially composed by each $\mathbf{A}_{\mathrm{b}}$ assembled along its main diagonal, then swap each pair of columns that correspond to the overlaid nodes (Fig. 2b) and remove the redundant column in the pair as well as the corresponding row with only zero elements to produce the final form of load distribution matrix $\mathbf{A}_{\mathrm{u}}$ for a basic unit. See Fig. 3, for more details please refer to literature [11].

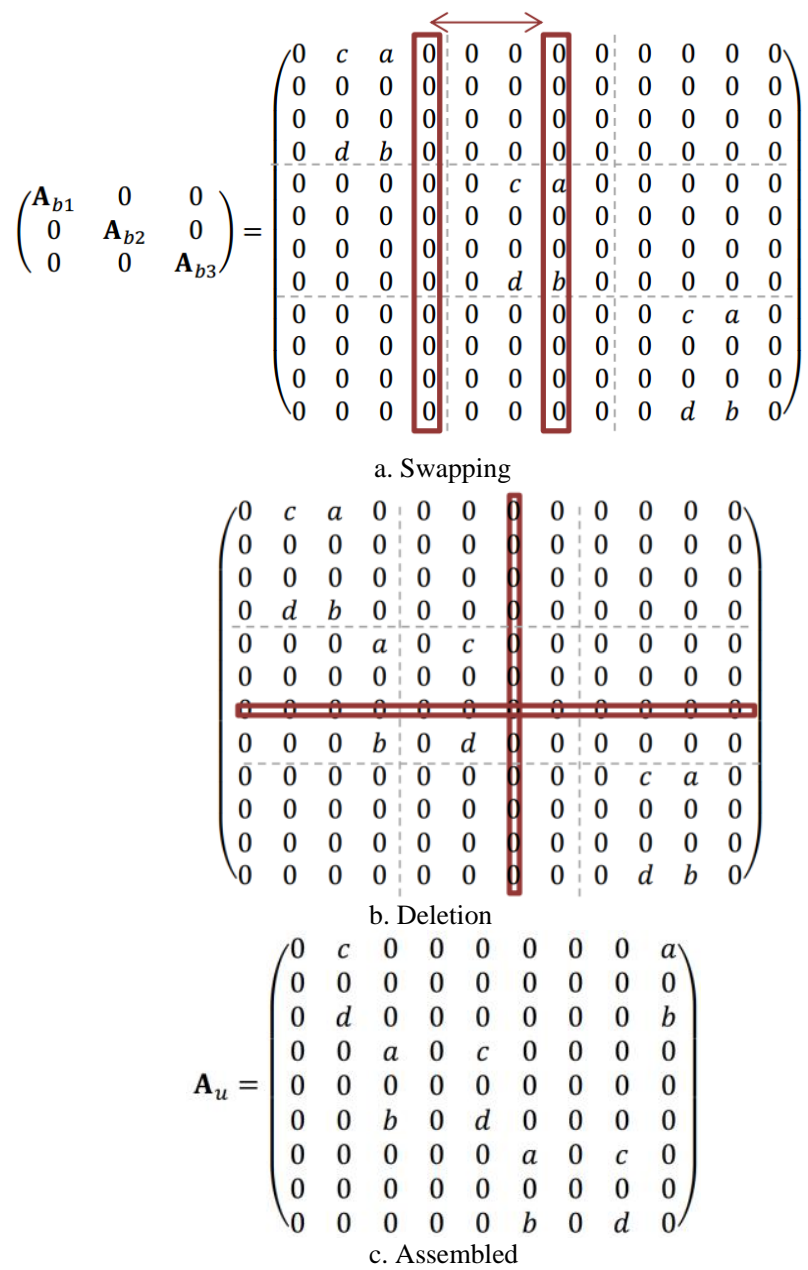

Fig. 3. Assembly matrix of a basic unit.

Then the nodal force of the basic unit can be calculated by $\mathbf{F}=\left(\mathbf{I}-\mathbf{A}_{u}\right)^{-1} \mathbf{F}_{0}$. Theoretically, by the same method it is also feasible to construct the matrix for the entire structure, which usually consists of tens or even hundreds of basic units. However, it would be labor consuming to complete the repetitive swap and removal of columns and rows in the matrix assembly. In order to perform the matrix assembly from basic units to the whole structure more efficiently, a standard matrix format inspired by Parigi, Sassone and Napoli [13] for basic units here is now developed. 


\section{STANDARD ASSEMBLY}

\section{A. Standard Assembly Format}

In general, the application of standard matrix of basic units transforms the matrix assembly process into matrix summation, a faster operation especially for large-scale reciprocal structures. The prerequisite, which is easily satisfied, is that the reciprocal system can be arranged as $m$ rows multiply $n$ columns of basic units. Specifically, repetitive reciprocal structures are of this pattern, an example can be found in [13], some others are displayed in Fig. 4. Note that the whole structure is decomposable into the same basic units.
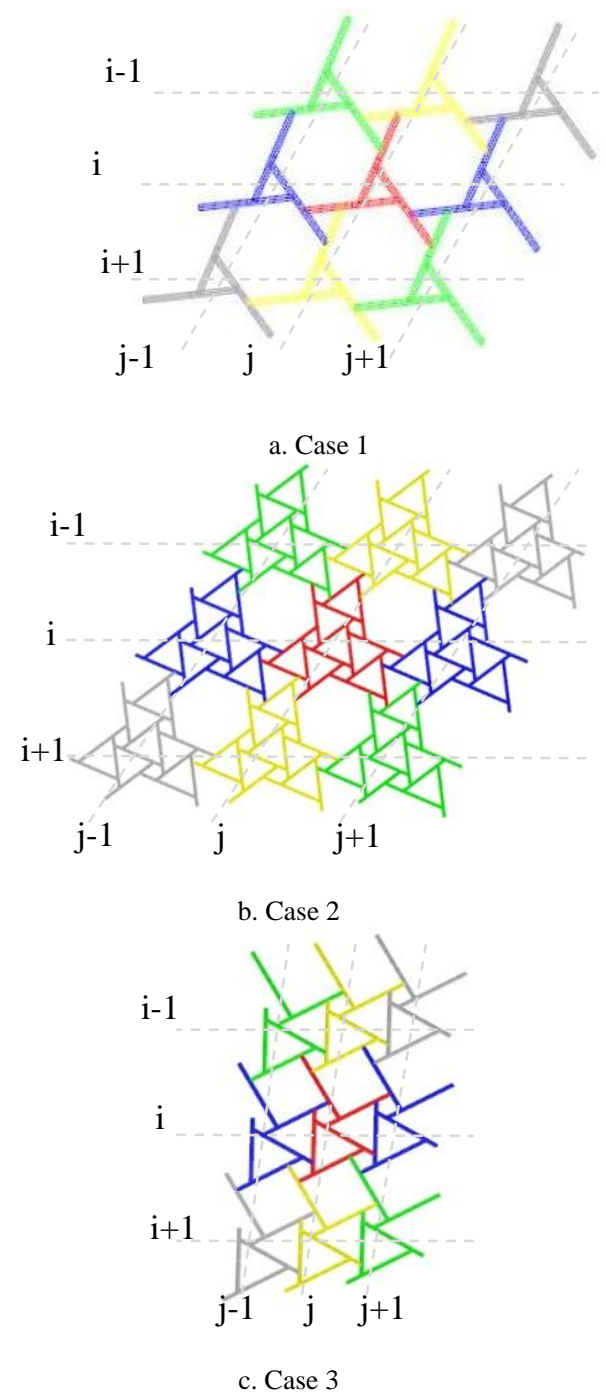

Fig. 4. Examples of different reciprocal types.

The idea is to adapt the basic unit matrix to the form defined by the relationship between a unit and its neighboring units so that every unit matrix can employ the same format and make slight changes according to its linkage variation with those around. For example, a basic unit at position $(i, j)$ in Fig. $4 a$ is connected to six other units around, i.e. the units at $(i, j-1),(i, j+1),(i-1, j),(i+1, j),(i-1, j-1)$ $(i+1, j+1)$. Enlightened by Parigi, Sassone and Napoli [13], a standard matrix form is constructed based on the unit at position (i,j) as

$$
\left(\begin{array}{c}
{[\mathbf{i}, \mathbf{j}-\mathbf{1}]} \\
{[\mathbf{0}]_{\mathbf{1}}} \\
{[0]_{2}} \\
{[\mathbf{i}-\mathbf{1}, \mathbf{j}-\mathbf{1}]} \\
{[\mathbf{0}]_{3}} \\
{[\mathbf{i n t e r n a l}]} \\
{[\mathbf{0}]_{3}} \\
{[\mathbf{i}+\mathbf{1}, \mathbf{j}]} \\
{[\mathbf{0}]_{2}} \\
{[\mathbf{i}+\mathbf{1}, \mathbf{j}+\mathbf{1}]} \\
{[\mathbf{i}, \mathbf{j}+\mathbf{1}]} \\
{[\mathbf{i}-\mathbf{1}, \mathbf{j}]}
\end{array}\right)
$$

As four nodes have been defined on each single bar to reveal the interaction among different bars, one row or a group of rows in this basic unit matrix accordingly corresponds to the nodes that have load transmission to other units. Every sub-matrix with position marker inside represents the node(s), within the basic unit, connected to the surrounding position. For instance, $[\mathbf{i}, \mathbf{j}-\mathbf{1}]$ means the sub-matrix related to the node(s) of unit at $(i, j)$ linked to the unit at (i,j-1) and it should be put in the top position of the basic unit matrix. Besides, there is [internal] describing the sub-matrix in relation to nodes with no connection to other basic units and [0] with all zero elements only to gap other sub-matrices for compatible assembly.

There are some quantitative constraints regarding the dimension, denoted as $d$ which describes how many nodes are involved, of these sub-matrices due to the repetitive characteristic. Because the linkage of unit $(i, j)$ with unit $(i, j+1)$ is identical to, when taking the current unit $(i, j+1)$ as the next unit $(i, j)$, the linkage of unit $(i, j)$ with unit $(i, j-1)$, the dimension of $[\mathbf{i}, \mathbf{j}-\mathbf{1}]$ and $[\mathbf{i}, \mathbf{j}+\mathbf{1}]$ should be the same, written as

$$
d_{i, j-1}=d_{i, j+1} \text {. }
$$

Similarly, there are

$$
\begin{gathered}
d_{01}=d_{i-1, j}=d_{i+1, j}, \\
d_{i-1, j-1}=d_{i+1, j+1}, \\
d_{i n}=d_{i, j-1}+d_{01}+d_{02}+d_{i-1, j-1}, \\
=d_{02}+d_{i+1, j+1}+d_{i, j+1}+d_{i-1, j} \\
d_{03}=(\mathrm{m}-1) d_{i n} .
\end{gathered}
$$

Here $d_{\text {in }}$ is the dimension of [internal], $d_{01}$ that of $[\mathbf{0}]_{1}$ and so on, $m$ is the number of rows of $m \times n$ arranged units. If we further make some denotations as following

$$
\begin{gathered}
a_{1}=d_{i n}, \\
a_{2}=a_{1}+d_{01}, \\
a_{3}=a_{1}-d_{i-1, j-1}-d_{02}=d_{i, j+1}+d_{i-1, j} .
\end{gathered}
$$


Then the dimension of a basic unit matrix is expressed as

$$
d_{\text {unit }}=\left(a_{1}+d_{\text {in }}\right) m+a_{2}=2 a_{1} m+a_{2} .
$$

The dimension of the matrix for the whole system is

$$
d_{\text {system }}=a_{1}(m-1)+\left(d_{\text {unit }}-a_{3}\right) n+a_{3} .
$$

Additionally, the row position of the first line of submatrix for unit at $(\mathrm{i}, \mathrm{j})$ in the matrix of the whole system is calculated by $H_{i, j}$, and

$$
H_{i, j}=a_{1}(m-i)+\left(d_{\text {unit }}-a_{3}\right)(j-1)+1 \text {. }
$$

\section{B. Case study}

To illustrate how the standard format works, a case with $2 \times 2$ three-bar units(Fig. 5a) are taken into account and the nodes are numbered in a basic unit as shown in Fig. 5b. It is obvious that

$$
m=n=2 \text {. }
$$

The standard relationship of the basic unit in the $2 \times 2$ system should refer to Fig. 4a, which shows the unit at $(i, j)$ has one node overlaying with every surrounding unit respectively and three internal nodes, so there are

$$
\begin{gathered}
a_{1}=d_{i n}, \\
d_{i-1, j-1}=d_{i+1, j+1}=d_{i-1, j}=d_{i+1, j}=d_{i, j-1}=d_{i, j+1}=1 .
\end{gathered}
$$

Substituting (15) (17) and (4) (8) into (9) (11), the following result can be obtained:

$$
a_{1}=3 ; a_{2}=4 ; a_{3}=2 ; d_{03}=3 \text {. }
$$

The dimensions of the basic unit and the whole structure are further acquired,

$$
d_{\text {unit }}=16 ; d_{\text {system }}=33
$$

When the standard format (3) applies to load vector of a basic unit, for example unit at $(1,1)$, the specific form is

$$
\begin{aligned}
\mathbf{F}_{1,1,0}= & \left(\begin{array}{llllllll}
F_{1,1}^{1} & 0 & F_{1,1}^{8} & {[\mathbf{0}]_{1 \times 3}} & F_{1,1}^{4} & F_{1,1}^{5} & F_{1,1}^{6} & {[\mathbf{0}]_{1 \times 3}} \\
& F_{1,1}^{7} & F_{1,1}^{3} & F_{1,1}^{9} & F_{1,1}^{2}
\end{array}\right)^{\mathrm{T}}
\end{aligned}
$$

The first two subscript numbers of $\mathbf{F}_{1,1,0}$ indicate the position of the unit and the third number zero means this is initial load. Inside the vector, superscript represents the node number of the unit. Since the system has a dimension of 33, the load vector with dimension of 16 should be expanded to the same dimension with zeros filling in the blank space. The first element of this load vector of the basic unit locates at row $H_{1,1}$ of the entire load vector,

$$
H_{1,1}=4 \text {. }
$$

Take $\mathbf{F}_{1,1,0}^{*}$ as the load vector after expansion,

$$
\begin{aligned}
& \mathbf{F}_{1,1,0}^{*}=\left(\left[\begin{array}{lllllllll}
{[\mathbf{0}]_{1 \times 3}} & F_{1,1}^{1} & 0 & F_{1,1}^{8} & {[\mathbf{0}]_{1 \times 3}} & F_{1,1}^{4} & F_{1,1}^{5} & F_{1,1}^{6} & {[\mathbf{0}]_{1 \times 3} .}
\end{array} .\right.\right. \\
& \left.\begin{array}{lllll}
F_{1,1}^{7} & F_{1,1}^{3} & F_{1,1}^{9} & F_{1,1}^{2} & {[\mathbf{0}]_{1 \times 14}}
\end{array}\right)^{\mathrm{T}}
\end{aligned}
$$

Finally the initial load vector for the system is (Fig. 6)

$$
\mathbf{F}_{0}=\mathbf{F}_{1,1,0}^{*}+\mathbf{F}_{1,2,0}^{*}+\mathbf{F}_{2,1,0}^{*}+\mathbf{F}_{2,2,0}^{*}
$$

Therefore, the initial load vector in $m \times n$ system can eventually be assembled as

$$
\mathbf{F}_{0}=\sum_{i=1}^{m} \sum_{j=1}^{n} \mathbf{F}_{i, j, 0}^{*}
$$

When the standard format is applied to load distribution matrix of a basic unit, rows of the matrix need to be adjusted simultaneously with columns in accordance with load vector, hence the matrix is expanded in a two dimensional manner. Corresponding to $\mathbf{F}_{1,1,0}, \mathbf{A}_{\mathrm{u}}$ is modified as

$\mathbf{A}_{1,1}=$
$\left(\begin{array}{llllllllllllllll}0 & 0 & 0 & 0 & 0 & 0 & a & 0 & 0 & 0 & 0 & 0 & c & 0 & 0 & 0 \\ 0 & 0 & 0 & 0 & 0 & 0 & 0 & 0 & 0 & 0 & 0 & 0 & 0 & 0 & 0 & 0 \\ 0 & 0 & 0 & 0 & 0 & 0 & 0 & 0 & 0 & 0 & 0 & 0 & 0 & 0 & 0 & 0 \\ 0 & 0 & 0 & 0 & 0 & 0 & 0 & 0 & 0 & 0 & 0 & 0 & 0 & 0 & 0 & 0 \\ 0 & 0 & 0 & 0 & 0 & 0 & 0 & 0 & 0 & 0 & 0 & 0 & 0 & 0 & 0 & 0 \\ 0 & 0 & 0 & 0 & 0 & 0 & 0 & 0 & 0 & 0 & 0 & 0 & 0 & 0 & 0 & 0 \\ 0 & 0 & d & 0 & 0 & 0 & 0 & b & 0 & 0 & 0 & 0 & 0 & 0 & 0 & 0 \\ 0 & 0 & 0 & 0 & 0 & 0 & 0 & 0 & b & 0 & 0 & 0 & 0 & 0 & d & 0 \\ 0 & 0 & 0 & 0 & 0 & 0 & b & 0 & 0 & 0 & 0 & 0 & d & 0 & 0 & 0 \\ 0 & 0 & 0 & 0 & 0 & 0 & 0 & 0 & 0 & 0 & 0 & 0 & 0 & 0 & 0 & 0 \\ 0 & 0 & 0 & 0 & 0 & 0 & 0 & 0 & 0 & 0 & 0 & 0 & 0 & 0 & 0 & 0 \\ 0 & 0 & 0 & 0 & 0 & 0 & 0 & 0 & 0 & 0 & 0 & 0 & 0 & 0 & 0 & 0 \\ 0 & 0 & 0 & 0 & 0 & 0 & 0 & 0 & 0 & 0 & 0 & 0 & 0 & 0 & 0 & 0 \\ 0 & 0 & 0 & 0 & 0 & 0 & 0 & 0 & a & 0 & 0 & 0 & 0 & 0 & c & 0 \\ 0 & 0 & 0 & 0 & 0 & 0 & 0 & 0 & 0 & 0 & 0 & 0 & 0 & 0 & 0 & 0 \\ 0 & 0 & c & 0 & 0 & 0 & 0 & a & 0 & 0 & 0 & 0 & 0 & 0 & 0 & 0\end{array}\right)$

which is a $16 \times 16$ matrix and its first element position (row position $H$ and column position $V$ ) in the whole matrix is

$$
H_{1,1}=V_{1,1}=4
$$

It should then be expanded to $32 \times 32$ by filling zeros in both columns and rows, and the matrix is denoted as $\mathbf{A}_{1,1}^{*}$. The Whole matrix for the entire structure is (Fig. 7) 


$$
\mathbf{A}=\mathbf{A}_{1,1}^{*}+\mathbf{A}_{1,2}^{*}+\mathbf{A}_{2,1}^{*}+\mathbf{A}_{2,2}^{*}
$$

Accordingly, the load distribution matrix for an $m \times n$ system is

$$
\mathbf{A}=\sum_{i=1}^{m} \sum_{j=1}^{n} \mathbf{A}_{i, j}^{*}
$$

In summary, there are three steps to obtain the whole matrix or vector for nodal force calculation:

1) Rearrangement of the load vector, building load distribution matrix for each basic unit;

2) Dimension expansion to agree with the dimension of the system;

3) Summation of matrix or vector of basic units

After the matrix $\mathbf{F}_{0}$ and $\mathbf{A}$ obtained for the entire structure, all nodal forces are immediately derived by (2). Post processing can be done to eliminate those nodes that are not in support or overlaying nodes, such as node 8 of unit at $(1,1)$, to simplify the final force vector.

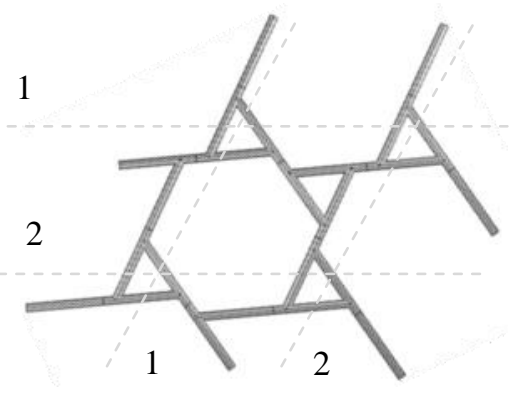

a. Assembly of basic units

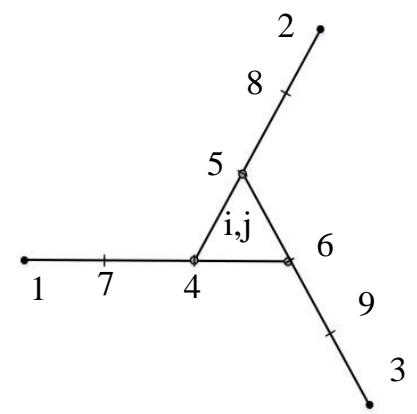

b. Numbering of a basic unit

Fig. 5. $2 \times 2$ reciprocal system.

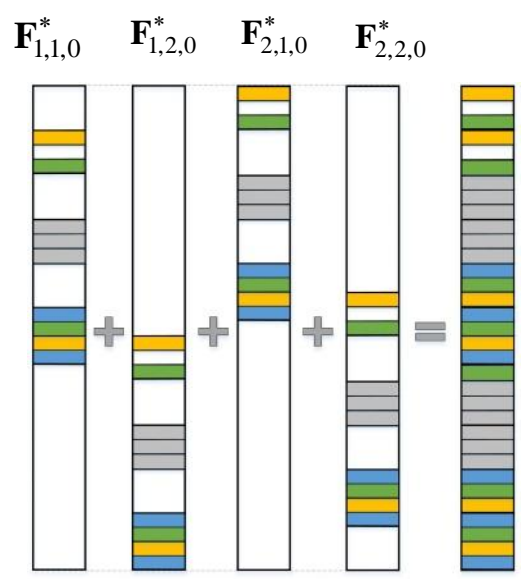

Fig. 6. Load vector assembly.

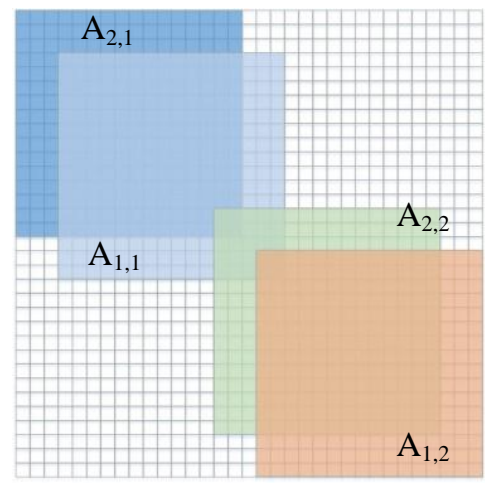

Fig. 7. Load distribution matrix assembly.

\section{VALIDATION}

To validate the effectiveness of the assembly, reaction forces under loading are compared between analytical formulation and FEM results (SAP2000 was employed for the finite element calculation). Given each bar in Fig. 5a the length of $1 \mathrm{~m}$ and is applied with uniform distributed load of $1 \mathrm{kN} / \mathrm{m}$, which is equivalent to $0.5 \mathrm{kN}$ at two ends of a bar when solving its nodal forces. The system has only pin joints and thus is statically determinate under perpendicular loading. Accordingly its internal force is irrelevant of material and bar section but relevant to its topological and geometrical property. Assuming that $a=d=0.4, b=c=0.6$, and (i,j) (k) represents the position of a unit and the node number inside that unit. The comparison between results from the proposed method and FEM is shown in Table I, it can be seen the reaction forces by two methods are exactly the same, which validates the correctness of the assembly process proposed.

TABLE I: COMPARISON OF REACTION FORCES BY FORMULATION AND FEM

\begin{tabular}{ccc}
\hline \hline Support position & Formulation $(\mathrm{kN})$ & SAP2000 $(\mathrm{kN})$ \\
\hline$(1,1)(1)$ & 2.30 & 2.30 \\
$(1,1)(2)$ & 1.83 & 1.83 \\
$(1,2)(2)$ & 1.41 & 1.41 \\
$(1,2)(3)$ & 1.68 & 1.68 \\
$(2,2)(3)$ & 1.42 & 1.42 \\
$(2,1)(3)$ & 2.15 & 2.15 \\
$(2,1)(1)$ & 1.21 & 1.21 \\
\hline \hline
\end{tabular}

\section{CONCLUSION}

A standard matrix format for basic reciprocal units is proposed to facilitate static analysis of different planar reciprocal systems. The format can be further adapted depending on different conditions. For load vector the format is applied in one dimensional way and as for load distribution matrix it extends to a two dimensional manner. Mathematical relationships are established for the determination of vector or matrix size and location of a basic unit as well as those of vector or matrix in a whole system, therefore vector or matrix in internal force calculation for an entire structure can be easily derived by summation. The proposed matrix assembly method, which efficiently solved the difficulty of static analysis of largescale reciprocal structures, can be used in any planar 
reciprocal structure with arrangement of $m$ rows and $n$ columns of repetitive basic units.

Future work would still focus on analytical formulation of spatial reciprocal structures where our standard matrix format is also workable since topological relationship between structural elements remains unchanged from $2 \mathrm{D}$ to 3D shape.

\section{REFERENCES}

[1] D. Yeomans, "The serlio floor and its derivations," Architectural Research Quarterly, vol. 2, pp. 74-83, 1997.

[2] M. Brocato and L. Mondardini, "Parametric analysis of structure from flat vaults to reciprocal grids," International Journal of Solids and Structures, vol. 54, pp. 50-65, 2015.

[3] O. Baverel, H. Nooshin and Y. Kuroiwa, "Configuration processing of nexorades using genetic algorithms," Journal of the International Association for Shell and Spatial Structures. vol. 45, pp. 99-108, 2004

[4] C. Douthe and O. Baverel, "Design of nexorades or reciprocal frame systems with the dynamic relaxation method," Computers and Structures. vol. 87, no. 21-22, pp.1296-1307, 2009.

[5] O. Baverel and A. Pugnale, "Reciprocal systems based on planar elements: Morphology and design explorations," Nexus Network Journal. vol. 16, no. 1, pp.179-189, 2014

[6] O. Popvic, J. C. Chilton, and B. S. Choo, "The variety of reciprocal frame (RF) morphologies developed for a medium span assembly building a case study," Journal of the International Association for Shell and Spatial Structures. vol. 39, no. 126, 1998.

[7] A. Pugnale and M. Sassone, "Structural reciprocity: Critical overview and promising research/design issues," Nexus Network Journal, vol. 16, no. 1, pp. 9-35, 2014

[8] J. Sánchez and F. Escrig, "Frames designed by leonardo with short pieces, An analytical approach," International Journal of Space Structures, vol. 4, no. 26, pp. 289 302, 2011.

[9] C. Douthe and O. Baverel, "Morphological and mechanical investigation of double-layer reciprocal structures," Nexus Network Journal, vol. 16, no. 1, pp. 191-206, 2014.
[10] S. Gelez, S. Aubry and B. Vaudeville, "Nexorade or reciprocal frame system applied to the design and construction of a $850 \mathrm{~m}^{2}$ archaeological shelter," International Journal of Space Structures, vol. 26, no. 4, pp. 303-311, 2011

[11] T. Kohlhammer and T. Kotnik, "Systemic behaviour of plane reciprocal frame structures," Structural Engineering International, vol. 21, no. 1, pp. 80-86, 2011

[12] S. Gelez, S. Aubry, and B. Vaudeville, "Behavior of a simple nexorade or reciprocal frame system," International Journal of Space Structures, vol. 26, no. 4, pp. 331 342, 2011.

[13] D. Parigi, M. Sassone and P. Napoli, "Kinematic and static analysis of plane reciprocal frames," in Proc. the International Association for Shell and Spatial Structures (IASS) Symposium.

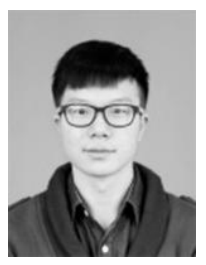

Ding Zou was a postgraduate in the College of Civil Engineering and Architecture, Zhejiang University, China from 2013 to 2016. His research focused on analysis and design of spatial structures and reciprocal structures.

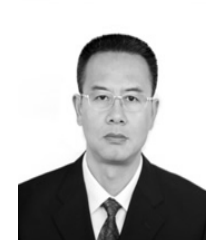

Nan Xiao is an associate professor in Zhejiang University, China. His principal research interests include: structural reliability analysis, seismic analysis and design of spatial structures, structural strengthening for existing buildings, etc. 